\title{
El ingeniero de inclusión y el lenguaje Scratch en el aprendizaje de la matemática
}

\author{
Jaime M. Cabrera-Medina (1), Irlesa I. Sánchez-Medina ${ }^{(1)^{*}}$ y Ferley Medina-Rojas ${ }^{(2)}$ \\ (1) Facultad de Ingeniería, Universidad Cooperativa de Colombia, Neiva, Colombia \\ (correo-e: jaime.cabrera@campusucc.edu.co; irlesa.sanchez@campusucc.edu.co). \\ (2) Facultad de Ingeniería, Universidad Surcolombiana, Neiva, Colombia (correo-e: ferley.medina@usco.edu.co). \\ * Autor a quien debe ser dirigida la correspondencia.
}

Recibido Jun. 26, 2020; Aceptado Ago. 26, 2020; Versión final Oct. 16, 2020, Publicado Dic. 2020

\begin{abstract}
Resumen
Se crea una estrategia didáctica mediada con Scratch apoyada con metodología STEAM. Se realiza una programación visual en temas ambientales con estudiantes de grado 9, se interviene con un programa de formación, con la aplicación de unas pruebas. El ingeniero de inclusión contribuye con la educación básica secundaria en el desarrollo de una estrategia didáctica para el fortalecimiento de las competencias matemáticas de razonamiento y argumentación, comunicación, representación y modelación, planteamiento y resolución de problemas. Los jóvenes investigadores del programa de ingeniería de sistemas asumen el rol de ingeniero de inclusión, y desarrollan una investigación mixta. El pretest obtiene un desempeño mínimo de 275 puntos, y el postest con 369 puntos, ubicada en un nivel de desempeño satisfactorio. El éxito radica que el ingeniero de inclusión conoce las dificultades presentes para trazar los objetivos de aprendizaje con procesos innovadores.
\end{abstract}

Palabras clave: ingeniero de inclusión; videojuegos; Scratch; competencias; STEAM

\section{The inclusion engineer and the Scratch language for the learning mathematics}

\begin{abstract}
A didactic strategy mediated with Scratch supported with STEAM methodology is created. Visual programming is carried out on environmental issues with grade 9 students. A training program is intervened with the application of some tests. The inclusion engineer contributes to basic secondary education in the development of a didactic strategy to strengthen the mathematical competencies of reasoning and argumentation, communication, representation, and modeling, posing, and solving problems. Young researchers from the systems engineering program assume the role of inclusion engineer, develop a mixed investigation. The pretest obtains a minimum performance of 275 points, and the posttest with 369 points, located at a satisfactory performance level. The success lies in the fact that the inclusion engineer knows the difficulties present in setting the learning objectives with innovative processes.
\end{abstract}

Keywords: inclusion engineer; video games; scratch; competence; STEAM 


\section{INTRODUCCIÓN}

Las instituciones de educación superior se articulan en proceso académicos, de la formación básica y media con actividades de proyección social e inclusión que garantice una educación de calidad durante el proceso de enseñanza aprendizaje desde cualquier contexto. Los estudiantes del programa ingeniería de sistemas se involucran con la educación básica secundaria para promover la ciencia, técnica, tecnología beneficiando a estudiantes de grado 9 en el desarrollo de las competencias matemáticas, utilizando la programación visual y asistida. En el presente siglo, es una necesidad el desarrollo de las habilidades matemáticas básicas enfocadas desde el saber, el hacer y el ser para que un individuo pueda desempeñarse de manera flexible y eficaz en las actividades diarias de este mundo globalizado.

Las comunidades educativas deciden apostar a la educación como eje que desarrolla las naciones, promueve la dotación de aulas, capacitación del personal docente acorde a los avances tecnológicos de la época que propicien aprendizajes activos, donde el protagonista es el estudiante y no el profesor. La Organización de las Naciones Unidas aprueba la Convención de los Derechos del Niño, considerando a niños y niñas como sujetos de derechos, con derecho a la educación, fundamentada en el desarrollo social, con accesibilidad, inclusión educativa basada en derechos humanos y educación de calidad (Vásquez, 2015).

Según (Loaiza e Hincapié, 2016) Colombia es considerado uno de los países con bajo nivel de calidad educativa y desigualdad desde cada una de sus regiones, de acuerdo a las pruebas PISA y Saber 11. Los jóvenes de 15 años tienen una capacidad para entender un texto (47\%), desarrollo de resultados científicos sencillos con su cotidianidad (55\%) e incapacidad de realizar inferencias básicas a partir de resultados matemáticos (71\%). Al comparar con países desarrollados de la Organización para la Cooperación y el Desarrollo Económicos (OCDE), se obtiene un promedio de calificación de 2 en una escala de 1 a 6, siendo evidente la importancia de inversión en el sector educación como un cambio en la forma de orientar la educación. Es importante que la educación superior se involucre en un modelo de responsabilidad social para contribuir a la transformación, impactando con estándares de calidad en el proceso educativo, mediada por infraestructuras tecnológicas blandas o duras, presentes en las instituciones educativas articuladas en la educación básica secundaria desde el concepto de equidad e inclusión en la educación superior apoyando los procesos educativos.

Para (Sánchez et al., 2015), consideran que las debilidades en el aprendizaje de las matemáticas radica en pocas estrategias o nulas, que involucren herramientas innovadoras para la promoción del conocimiento. Es aquí, donde los estudiantes del programa ingeniería de sistemas (Ingeniero de inclusión) tienen presente el diseño de videojuegos ayudando a los niños y jóvenes a aprender a aprender por sí mismos, con la idea que al utilizar videojuegos los niños aprenden un nuevo alfabetismo, por este motivo se utiliza Scratch como herramienta de software para la creación de juegos en la educación básica secundaria. Según (Küster y Castillo 2012), el videojuego permite controlar la ansiedad, el aburrimiento, miedo, enfado, distracción, alegría, y motivación, involucrando los conceptos de programación. (Rueda y Franco, 2018), presentan que la comunidad educativa en Colombia es testigo de la incorporación de las tecnologías en las escuelas, el campo de la "Tecnología e Informática" aparece como un "área obligatoria y fundamental de la educación básica" en la Ley 115 de 1994 y su incorporación al currículo se estableció por medio de la Resolución 2343 de 1996, inscrita en un proceso de concertación entre el Ministerio de Educación Nacional (MEN) y las federaciones y confederaciones de educadores de los sectores público y privado.

Scratch (Vidal et al.,2015) en el aprendizaje de las matemáticas describe la construcción de algoritmos y elementos (Marimuthu y Govender, 2018), presenta el diseño de actividades interactivas de estudiantes que aprendieron las operaciones básicas desarrollando su creatividad, y la solución de problemas (Rodríguez et al.,2020), (Acuña, 2018), (Ouahbi et al., 2015), el máximo común divisor (Baeza et al., 2017), apoyo al aprendizaje en estudiantes de primaria (Dapozo et al., 2016), la programación de robots (Scherer et al., 2020). Una estrategia didácticas para el aula es la construcción de un videojuego educativo que se puede considerar como un objeto virtual de aprendizaje que promueve la adquisición de conocimientos de forma autodidacta con ayuda de las Tecnologías de la Información y la Comunicación (TIC) (Cabrera et al., 2016).

Existen categorías de actividades de aprendizaje para que el estudiante utilice las herramientas que involucra el software educativo (Kordaki, 2012). El déficit de ingenieros radica en el poco interés por estudiar el área de las ciencias básicas fundamentadas en las matemáticas, como se evidencia en los antecedentes y las investigaciones en educación básica y media relacionadas a estrategias, métodos, procedimientos, para adquirir las bases de las matemáticas, y garantizar futuros profesionales de Ingeniería. El ingeniero de inclusión fortalece la competencia requerida en las ciencias básicas al ayudar a otros estudiantes, para este caso de grado 9 en la estrategia didáctica al participar y aprender las matemáticas con Scratch. 
Ser ingeniero conlleva a utilizar el ingenio para desarrollar una actividad manual, uniendo la ciencia, técnica y tecnología para la resolución de problemas con mucha ética. La responsabilidad social será una obligación como integrante de una comunidad, es aquí donde un título profesional compromete a un individuo con su contexto promoviendo buenas prácticas en beneficio de una mejorar calidad de vida. Por otra parte, la inclusión social busca resolver problemas, posiblemente del día a día para un cambio de hábitos, costumbres, hasta lograr satisfacer necesidades, involucrando individuos para alcanzar el objetivo propuesto en la solución de la dificultad presente. El futuro profesional de la línea de ingeniería debe conocer los 17 Objetivos de Desarrollo Sostenible (ODS) para identificar los desafíos mundiales que permitirán acabar con la pobreza y promover la prosperidad económica, la inclusión social, la sostenibilidad ambiental, la paz y apoyar en el fortalecimiento de un buen gobierno (Girón, 2016). El ingeniero de inclusión contribuye en el desarrollo de habilidades matemáticas necesarias para que los individuos que habitan este mundo globalizado se pueda desempeñar sin angustia en cualquier entorno, profesional, cultural, social, académico (Monjelat, 2019). Además, será el encargado de planificar, diseñar, desarrollar, implementar y mantener sistemas de información para la toma de decisiones, apoyado de una cultura donde el factor fundamental es la ciencia, la técnica, la tecnología e innovación, para beneficiar cualquier contexto o comunidad que necesiten apoyo o dificultades presentes (Sánchez et al., 2015).

Existen juegos como Minecraft, intuitivo en versión Android y IOS (Hewett et al., 2020), El ScratchJr, se enfoca a un público infantil, entre 5 a 7 años, con una interfaz simple y sencilla, que cambia textos por iconos para ser entendida por usuarios aun analfabetos (Moreno et al., 2020), (Zhang y Nouri, 2019). De la experiencia adquirida con el manejo del software para el desarrollo de videojuegos, se puede resaltar que el uso del Storyboard para el diseño de videojuegos permite presentar una narrativa o guion mediante ilustraciones en secuencia, como guía de la historia a desarrollar en el juego, que será luego organizada como una animación estructurada en el software seleccionado (Pejman, 2014). Las competencias matemáticas necesitan de ambientes de aprendizaje enriquecidos por situaciones problema significativo y comprensivo, que posibiliten avanzar a niveles de competencia más complejos, como se presenta en la tabla 1.

Tabla 1: Competencias matemáticas en grado 9 (MEN, 2016)

\begin{tabular}{|l|l|l|}
\hline $\begin{array}{l}\text { Razonamiento y } \\
\text { argumentación }\end{array}$ & $\begin{array}{l}\text { Comunicación, representación y } \\
\text { modelación }\end{array}$ & Planteamiento y resolución de problemas \\
\hline $\begin{array}{l}\text { Percibir regularidades y } \\
\text { relaciones. Predicciones y y } \\
\text { conjeturas. }\end{array}$ & $\begin{array}{l}\text { Comunicar en distintos lenguajes lo } \\
\text { que lee y se escribe. } \\
\text { Explicar e interpretar con }\end{array}$ & $\begin{array}{l}\text { Desarrollar estrategias para resolver } \\
\text { propio de las matemáticas. } \\
\text { problemas. }\end{array}$ \\
$\begin{array}{l}\text { argumentos y razones. } \\
\text { Validar o invalidar } \\
\text { conclusiones con base en } \\
\text { modelos. }\end{array}$ & $\begin{array}{l}\text { Comunicar la resolución de } \\
\text { problemas matemáticos a través de } \\
\text { sistemas mentales, gráficos o } \\
\text { tridimensionales en formas }\end{array}$ & $\begin{array}{l}\text { nuevos problemas variando condiciones. } \\
\text { Resolver problemas aportando múltiples } \\
\text { soluciones. } \\
\text { esquemantear preguntas a partir de problemas } \\
\text { donde falta información o con textos narrativos } \\
\text { o incompletos. }\end{array}$ \\
\hline
\end{tabular}

Como parte de los componentes involucrados en la competencia para grado 9 se encuentra: 1) numéricovariacional pensamiento numérico centrado en la comprensión del uso y significado de los números, la numeración, comprendiendo el sentido y significado de las operaciones y relaciones entre números, desarrollando diferentes técnicas de cálculo y estimación. El pensamiento Variacional se desarrolla en estrecha relación con tipos de pensamiento matemático como el numérico, el espacial, el de medida o métrico y el aleatorio o probabilístico, y con otros tipos de pensamiento, aunque se representan usualmente por medio de sistemas algebraicos y analíticos, requieren de conceptos y procedimientos relacionados con distintos sistemas numéricos. 2) geométrico-métrico el pensamiento geométrico desde el arte, decoración, diseño y construcción de objetos artesanales y tecnológicos, en relación con la educación física, los deportes, la danza, observación, reproducción de patrones, con formas de lectura y comprensión del espacio, elaboración e interpretación de mapas, representaciones a escala de sitios o regiones en dibujos y maquetas, entre otras situaciones (Calder, 2019). El pensamiento métrico, centrado en la construcción de los conceptos de magnitud, comprensión de los procesos de conservación de magnitudes, estimación de la medida de cantidades de distintas magnitudes y los aspectos del proceso de "capturar lo continuo con lo discreto", apreciación del rango de las magnitudes, selección de unidades de medida, de patrones y de instrumentos y procesos de medición, diferencia entre la unidad y los patrones de medición, asignación numérica y el papel del trasfondo social de la medición. 3) aleatorio este pensamiento es apoyado en conceptos y procedimientos de la teoría de probabilidades, estadística inferencial, estadística descriptiva, en la combinatoria, buscando soluciones razonables a problemas en los que no hay una solución clara y segura, con un espíritu de exploración y de investigación mediante la construcción de modelos de fenómenos físicos, sociales o de juegos de azar y uso de estrategias como la exploración de sistemas de datos, simulación de experimentos y la realización de conteos (MEN, 2016). 
STEAM (Science, Technology, Engineering, Art, Mathematics) es la sigla de la metodología educativa que busca educar a los estudiantes en cinco disciplinas específicas (Ciencia, Tecnología, Ingeniería, Arte y Matemáticas), fomentando la concentración en prácticas, habilidades para el aprendizaje, construcción de modelos físicos, biológicos, computacionales y matemáticos como base para la formación científica continua, interdisciplinar, el aprendizaje formal e informal, la investigación, logrando integración de saberes y conocimientos, teniendo presente la lúdica como factor de éxito. Los juguetes educativos y software para diseño de videojuegos engloban procesos de aprendizaje basados en resolución de problemas mediante el razonamiento lógico, incorporación de situaciones de la vida cotidiana, uso de herramientas tecnológicas y repetición de un hecho. Lo representativo de esta metodología STEAM para ser utilizada en el sistema educativo, es que los estudiantes realicen un trabajo en equipo, puedan tomar decisiones en conjunto, resolver problema de forma rápida y creativa, el incremento de la autoestima, desarrollar la capacidad comunicativa, el aprendizaje mediante la experimentación, desarrollo de la memoria al retener conceptos aprendidos, desarrollo de la imaginación e innovación al crear cosas nuevas, entre otros (Thuneberg et al., 2018). (Pérez et al. ,2019), la estrategia didáctica permite procedimientos y recursos utilizados por el docente para un aprendizaje significativo, facilitando el procesamiento del contenido nuevo de una forma profunda y consciente, donde el docente se apoya de un plan de acción flexible considerando procedimientos que el agente de enseñanza utiliza en forma reflexiva promoviendo el logro del aprendizaje significativo en estudiantes.

\section{METODOLOGÍA}

Los jóvenes investigadores del programa ingeniería de sistemas (ingeniero de inclusión) desarrollaron una investigación aplicada de tipo mixta. Una muestra de 34 estudiantes de educación básica secundaria grado 9 de una institución educativa oficial del departamento Huila. El objetivo trabajar una estrategia didáctica para el fortalecimiento de la competencia matemática mediante Scratch, desde el concepto de los videojuegos creando una programación visual, relacionada con temas ambientales. Proceso apoyado con la metodología STEAM como se muestra en la tabla 2, que enumera las competencias a desarrollar.

Tabla 2: Estrategia didáctica para el desarrollo de las competencias matemáticas propuesta por el ingeniero de inclusión

\begin{tabular}{|c|c|c|}
\hline \multirow[b]{2}{*}{ Herramienta } & \multicolumn{2}{|r|}{ Metodología STEAM } \\
\hline & $\begin{array}{c}\text { Área de } \\
\text { conocimiento }\end{array}$ & Competencias \\
\hline Scratch & Matemáticas & $\begin{array}{l}\text { Ciencia: cuidado y conservación del medio ambiente } \\
\text { Tecnología: herramientas de hardware y software. } \\
\text { Ingeniería: programación de video juego } \\
\text { Arte: Storyboard } \\
\text { Matemática: Razonamiento y argumentación; Comunicación, representación y } \\
\text { modelación; Planteamiento y resolución de problemas. }\end{array}$ \\
\hline
\end{tabular}

Fue necesario el diseño de instrumentos como técnicas de recolección de información, abordando las siguientes fases: 1) diagnóstica: Se realizó una investigación documental teniendo como fuentes la página del ICFES interactivo prueba ICFES saber 9, la plataforma del profesor Milton Ochoa prueba pensar 9, las pruebas por nivel de desempeño y acumulativas. También, los resultados de la observación directa del grupo en aula, presenta un desempeño crítico, el $13 \%$ de los estudiantes tenían un nivel de desempeño insuficiente, y el $57 \%$ en un nivel de desempeño mínimo. Indicando que los estudiantes de noveno grado poseen conocimientos básicos de matemáticas, no comprenden los procesos que se deben seguir para resolver los problemas que plantea la prueba. El objetivo de recuperar los logros perdidos durante el primer y segundo semestre académico no se alcanzan, en el primer semestre el $80 \%$ lo reprueban y para el segundo semestre un $77 \%$. Esto indica que los conceptos matemáticos orientados en el aula de clase no son adquiridos ni comprendidos por los estudiantes. Según el análisis anterior, se trabaja una categorización de variables a intervenir encontrando las siguientes: 1) variables competencias: C1: razonamiento y argumentación, C2: comunicación, representación y modelación, C3: planteamiento y resolución de problemas. 2) variables componentes: CO1: numérico-variación, CO2: geométrico-métrico, CO3: aleatorio.

Antes de aplicar la estrategia didáctica se diseña un instrumento como pretest, con las siguientes variables: $\mathrm{P} 1$ = escoger un modelo para el concepto de volumen y capacidad - eficiencia. P2 = lectura del plano cartesiano - ubicación espacial. P3 = resolución de ecuaciones - balanceo de una balanza. P4 = gráfica estadística - lectura y análisis de gráficas. P5 = planteamiento de ecuaciones a partir de una información. P6 = lectura de gráficos - análisis geométrico. P7 = lectura de diagramas de árbol - toma de decisiones. P8 = lectura de solidos geométricos - identificación de sus componentes. P9 = lectura de gráficas circulares manejo de porcentajes. P10 = lectura de gráficas - comportamiento físico de una partícula. Como parte de la validación del instrumento se encuentra un índice de confiabilidad del $51,3 \%$, y con un coeficiente de fidelidad del pretest del 1,00 según análisis de consistencia de Kuder y Richardson. 
2) de desarrollo: El ingeniero de inclusión, desarrolla la estrategia didáctica con metodología STEAM, en estudiantes de grado 9, utilizando Scratch como software para crear video juegos relacionados con el tema ambiental, encontrando programas visuales, donde el video juego evidencia acciones con objetos inmersos, relacionados con la ubicación espacial, análisis de gráficas, y geométrico, toma de decisiones, identificación de componentes, comportamientos, capacidades y cálculos. Para este logro, se realiza una intervención que permitió implementar un programa de capacitación con las siguientes etapas: 1) diseño de videojuegos (StoryBoard): a cada estudiante se le doto de papel, lápiz, marcadores, temperas, crayolas, colores, para plasmar en el papel la historia que pretendían desarrollar en Scratch como videojuego - motivo central el medio ambiente para algunos casos "reciclando plástico", siempre en cada caso se involucraron las competencias matemáticas. Además, cada estudiante expuso de forma oral su historieta dando claridad al nombre del videojuego, describe el escenario, y expresan el objetivo de creación. 2) desarrollo del videojuego: el ingeniero de inclusión desarrolla seis sesiones de cuatro horas de capacitación, sobre el uso de Scratch y la programación de videojuegos, en esta fase los estudiantes de grado 9 trabajaron en equipo en el computador para plasmar lo presentado en el StoryBoard. La lógica, creatividad, fueron utilizadas en la programación en bucles, bloques, como las competencias matemáticas.

3) implementación del videojuego: cada estudiante explico y compartió en la sala de cómputo el videojuego creado con sus compañeros de grupo, bajo la mirada atenta del ingeniero de inclusión y un grupo de expertos pedagogos e ingenieros. La evaluación se realizó según los criterios propuestos en cuanto a temas ambientales, que evidenciaban las competencias matemáticas desde el razonamiento, la argumentación, comunicación, interpretación, modelación, planteamiento y resolución de problemas, según las acciones presentadas en cada videojuego, los estudiantes luego se sometieron a la prueba postest.

4) reingeniería del videojuego: En esta fase cada estudiante programador de videojuego realizó los ajustes al producto desarrollado atendiendo las observaciones obtenidas en la fase de implementación por los expertos. Los videojuegos que presentaron el mayor número de competencias de forma sencilla y clara se subieron a la plataforma de la comunidad Scratch.

\section{RESULTADOS Y DISCUSIÓN}

A partir de los resultados del pretest se utilizan las herramientas de acuerdo con las competencia y componentes según la escala propuesta por el ICFES en su prueba saber 9, presentando niveles de desempeño como: 1) insuficiente: con una escala de puntaje de $100-235.2)$ mínimo: con una escala de puntaje de 236 - 315. 3) satisfactorio: con una escala de puntaje de $316-415$. 4) avanzado: con una escala de puntaje de 416 - 500. Luego, con los resultados de la fase diagnostica las variables de investigación a intervenir se continuo con la fase de desarrollo. Los estudiantes de grado 9 involucrados en la creación de los juegos con enfoque ambiental, pero con acciones que involucraban las competencias matemáticas con sus respectivos componentes, presentaron los diferentes videos juegos que se detallan en la tabla 3 . Cada uno, con el objetivo del juego que facilita el aprendizaje de la competencia en sus componentes como parte del diseño y desarrollo (programación - lógica matemática).

Durante el desarrollo de la estrategia didáctica utilizando Scratch como software para diseñar videojuegos desde el tema ambiental, pero aplicando acciones durante la programación de los videojuegos relacionados con cada una de las competencias matemáticas, se aplicó una prueba pretest y postest para determinar la competencia obtenida durante el desarrollo de la estrategia. En ella, se analiza que cada una de las preguntas tanto del pretest, como del postest, miden el nivel de dificultad de cada una de las preguntas relacionadas con las competencias y componentes matemáticos que se evalúan en el grado 9 . Se determinó en el pretest que ninguna pregunta es descartable, ni existieron preguntas fáciles en el momento de contestar por parte de los estudiantes. La fig. 1 da a conocer la competencia donde los estudiantes presentaron mayor grado de dificultad en el pretest corresponde a comunicación, representación y modelación con promedio porcentual del $39,20 \%$ de respuestas correctas y la competencia con menor dificultad corresponde a planteamiento y resolución de problemas con promedio de $51,00 \%$ de respuestas correctas.

Adicionalmente, se presenta la competencia de mayor grado de dificultad en el postest, corresponde a razonamiento y argumentación con un promedio porcentual del $45,60 \%$ de respuestas correctas y la competencia con menor dificulta es comunicación, representación y modelación con promedio de 58,80\% de respuestas correctas. Los estudiantes de grado 9 usan los videojuegos desarrollados en Scratch por ellos, al igual que el profesor en el aula como estrategia didáctica para apoyar procesos de aprendizaje. Estrategia efectiva porque se observa que el rendimiento porcentual en las tres competencias evaluadas tanto en pretest como es postest es superior en el postest. 
Tabla 3: Metodología STEAM en la creación del juego para la competencia matemática

\begin{tabular}{|c|c|c|c|c|}
\hline $\begin{array}{l}\text { Nombre del } \\
\text { juego }\end{array}$ & Objetivo del juego & Conocimiento & Competencias & Componente \\
\hline $\begin{array}{l}\text { El gato } \\
\text { cartesiano. }\end{array}$ & $\begin{array}{l}\text { Reconocer los } \\
\text { elementos de un } \\
\text { plano cartesiano } \\
\text { con el trazado y } \\
\text { ubicación de puntos } \\
\text { coordenados. }\end{array}$ & $\begin{array}{l}\text { Mediante el trazado del plano } \\
\text { cartesiano, se identifica } \\
\text { abscisas, ordenadas, } \\
\text { cuadrantes, y la ubicación de } \\
\text { los signos. }\end{array}$ & $\begin{array}{l}\text { Comunicación, } \\
\text { representación y } \\
\text { modelación. Razonamiento } \\
\text { y argumentación. } \\
\text { Planteamiento y resolución } \\
\text { de problemas. }\end{array}$ & $\begin{array}{l}\text { Numérico } \\
\text { variacional, } \\
\text { geométrico } \\
\text { métrico y } \\
\text { aleatorio. }\end{array}$ \\
\hline $\begin{array}{l}\text { El gato entre } \\
\text { parábolas. }\end{array}$ & $\begin{array}{l}\text { Reconocer el } \\
\text { gráfico de una } \\
\text { ecuación cuadrática } \\
\text { con su trazado en el } \\
\text { plano cartesiano. }\end{array}$ & $\begin{array}{l}\text { A partir de la ecuación } \\
A x^{2}+B x+C=0 \text {, se reconoce la } \\
\text { gráfica de una parábola y de } \\
\text { acuerdo con el signo del } \\
\text { coeficiente de } x^{2}(A) \text { identifica } \\
\text { su comportamiento. }\end{array}$ & $\begin{array}{l}\text { Comunicación, } \\
\text { representación y } \\
\text { modelación. Razonamiento } \\
\text { y argumentación. }\end{array}$ & $\begin{array}{l}\text { Numérico } \\
\text { variacional, } \\
\text { geométrico } \\
\text { métrico y } \\
\text { aleatorio. }\end{array}$ \\
\hline $\begin{array}{l}\text { El gato en el } \\
\text { laberinto. }\end{array}$ & $\begin{array}{l}\text { Identificar el uso } \\
\text { correcto de un kit } \\
\text { ecológico de } \\
\text { acuerdo con } \\
\text { colores. }\end{array}$ & $\begin{array}{l}\text { Aprende, reconoce y deposita } \\
\text { los residuos sólidos en los } \\
\text { recipientes de acuerdo con el } \\
\text { código de colores normativos } \\
\text { para un kit ecológico. }\end{array}$ & $\begin{array}{l}\text { Comunicación, } \\
\text { representación y } \\
\text { modelación. Razonamiento } \\
\text { y argumentación. } \\
\text { Planteamiento y resolución } \\
\text { de problemas. }\end{array}$ & $\begin{array}{l}\text { Numérico } \\
\text { variacional, } \\
\text { geométrico } \\
\text { métrico y } \\
\text { aleatorio. }\end{array}$ \\
\hline $\begin{array}{l}\text { El gato cuida } \\
\text { el medio } \\
\text { ambiente. }\end{array}$ & $\begin{array}{l}\text { Identificar los } \\
\text { residuos para su } \\
\text { disposición en las } \\
\text { bolsas de acuerdo } \\
\text { con los colores } \\
\text { normativos. }\end{array}$ & $\begin{array}{l}\text { Diferencia residuos plásticos } \\
\text { de los orgánicos, } \\
\text { depositándolos en sus } \\
\text { respectivas bolsas atendiendo } \\
\text { código de colores normativos. }\end{array}$ & $\begin{array}{l}\text { Comunicación, } \\
\text { representación y } \\
\text { modelación. Razonamiento } \\
\text { y argumentación. } \\
\text { Planteamiento y resolución } \\
\text { de problemas. }\end{array}$ & $\begin{array}{l}\text { Numérico } \\
\text { variacional, } \\
\text { geométrico } \\
\text { métrico y } \\
\text { aleatorio. }\end{array}$ \\
\hline
\end{tabular}

La fig. 1, para el caso de los componentes matemáticos evaluados donde los estudiantes presentan mayor grado de dificultad en el pretest corresponden al numérico - variacional y al aleatorio con promedio porcentual del $39,20 \%$ de respuestas correctas y el componente con menor dificulta corresponde al geométrico - métrico con promedio de $57,40 \%$ de respuestas correctas. Se destaca en la fig. 1, el componente matemático evaluado en el que los estudiantes presentan mayor grado de dificultad en el postest corresponde al numérico - variacional con promedio porcentual del $48,00 \%$ de respuestas correctas y el componente con menor dificulta corresponde al geométrico - métrico con promedio de $61,70 \%$ de respuestas correctas.

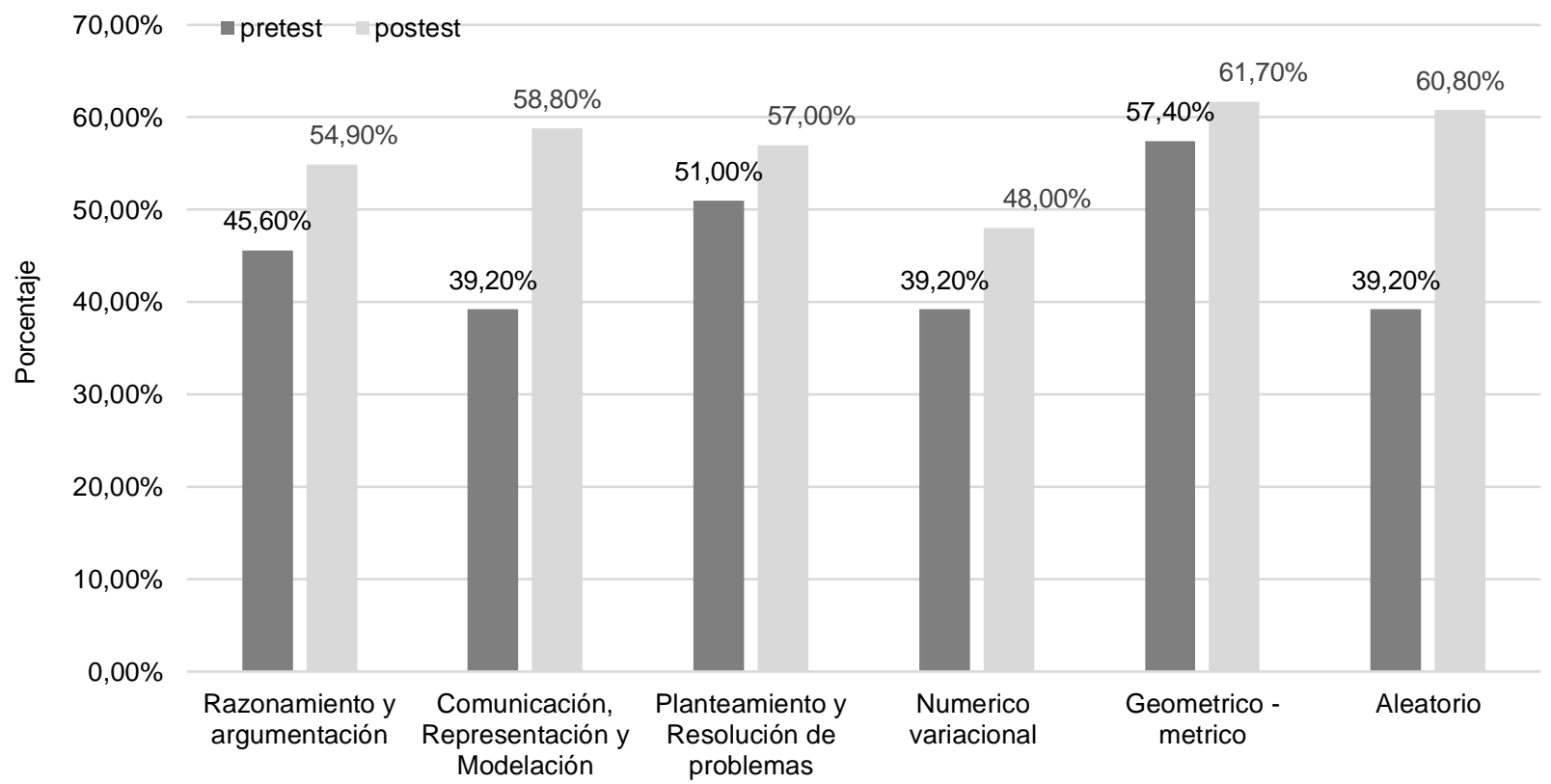

Fig. 1. Porcentaje rendimiento por competencia y componentes evaluados - comparativo pretest y postest

De igual forma en la fig. 1, se presenta en su conjunto que los estudiantes de grado 9 al hacer uso de los videojuegos desarrollados en Scratch por ellos mismo y utilizado por el profesor en el aula como estrategia didáctica para apoyar procesos de aprendizaje de aula fue muy efectivo porque se observa que el rendimiento porcentual en los tres componentes evaluados en el pretest es superior en el postest. Como resultado final el 
puntaje promedio del pretest (275 puntos) que corresponde a un nivel de desempeño minino es inferior al puntaje promedio del postest (369 puntos) que corresponde a un nivel de desempeño satisfactorio, con una diferencia entre ellos de 94 puntos. Además, la altura de la caja de evaluación pretest que corresponde a (375 puntos) permite asegurar que los puntajes que obtuvieron los estudiantes fueron muy heterogéneo comprendidos entre (275 a 500 puntos) presentando dos puntajes atípicos en 100 puntos, mientras que la altura de la caja evaluación postest que corresponde a ( 275 puntos) permite asegurar que los puntajes que obtuvieron los estudiantes fueron homogéneos comprendidos entre (225 a 500 puntos), se determina que la diferencia en alturas entre las cajas es de 100 puntos.

Con el resultado obtenido se confirma lo propuesto por (Baeza et al., 2017), que al involucrar una estrategia didáctica mediada con Scratch se logra un desarrollo de las matemáticas. De igual forma (Marimuthu y Govender, 2018), están de acuerdo que los video juegos desarrollan la lógica. Por otra parte (Rodríguez et al., 2020) afirman que también se desarrolla el pensamiento lógico matemático en el nivel básico. (Acuña, 2018) confirma la importancia de las competencias matemáticas al desarrollar la creatividad, solución de problemas y motivación por el aprendizaje de esta área. Adicionalmente, el ingeniero de inclusión se apropia de la metodología STEAM para que los estudiantes de grado noveno desarrollen videojuegos que promuevan las competencias matemáticas.

\section{CONCLUSIONES}

El uso de Scratch permite una mediación en el proceso de aprendizaje de conceptos matemáticos, el desarrollo de competencias, como estrategia para incrementar rendimientos académicos, motivando a cambios de paradigmas para orientar el desarrollo de una clase. El desarrollo de las habilidades matemáticas fomenta el pensamiento lógico, métodos para solucionar problemas, hábito de hacer autodiagnóstico con respecto a su trabajo, capacidad de poner en duda las ideas de uno mismo, posibilidad de obtener resultados complejos a partir de ideas simples, trabajar cada cual a su propio ritmo en función de sus propias competencias, aprender a asumir conceptos matemáticos: coordenadas, variables, algoritmos, aleatoriedad, aprender los fundamentos de la programación, usar distintos medios: sonido, imagen, texto, grafico, y posibilitar el aprendizaje colaborativo a través del intercambio de conocimiento.

Scratch es una excelente alternativa como estrategia didáctica para el fortalecimiento de falencias conceptuales presentes en matemática básica según las pruebas saber aplicadas en la Institución Educativa. Una estrategia didáctica, apoyada por videojuego, con una metodología STEAM, permite desde el tema ambiental desarrollar las competencias matemáticas. El razonamiento y argumentación, comunicación, representación y modelación, planteamiento y resolución de problemas, desde el trabajo en sus componentes numérico - variacional, geométrico - métrico y aleatorio, evidenciado por las mejoras representativas en la comparación de los instrumentos aplicados.

El ingeniero de inclusión contribuye a una educación de calidad en la educación básica secundaria al promover el desarrollo de competencias matemáticas mediante videojuegos diseñados con Scratch. Además, construye una estrategia didáctica utilizando la metodología STEAM para desarrollo del pensamiento crítico, la imaginación, la creatividad, resolución de problemas, que impactan las competencias matemáticas.

El éxito de un videojuego en educación matemática radica en el ingeniero de inclusión, que antes de llevarlo al aula debe conocerlo, debe haber jugado, este conocimiento le permitirá trazar objetivos de aprendizaje para hacer del proceso enseñanza - aprendizaje, un proceso diferente al tradicional, un proceso donde la motivación, la interactividad, el uso de las nuevas tecnologías de la información y la comunicación, como también el constante trabajo en equipo colaborativo con metodología STEAM, promueva el estudio de los conceptos que involucra la formación matemática del ser humano con el tratamiento de los problemas de aprendizaje y sus aplicaciones, la ubicación en el espacio - tiempo y la adquisición de valores ético - morales, potenciando así, la formación por competencia en el saber, en el hacer, y en el ser.

\section{REFERENCIAS}

Acuña, N., Aprendizajes de las Matemáticas Mediados por Juegos Interactivos en Scratch en la IEDGVCS, https://doi.org/10.17981/cultedusoc.9.2.2018.03, Rev. Cultura Educación y Sociedad, 9(2), 32-42, (2018).

Baeza, M. A., Claros, F. J., y otros dos autores, Pensamiento matemático avanzado y Scratch: el caso de máximo común divisor. Rev. de Investigación e Innovación Educativa "Pensamiento Matemático", 7(3), 43-64, (2017).

Cabrera, J. M., Sánchez, I. I., y Rojas, F., Uso de objetos virtuales de aprendizaje OVAS como estrategia de enseñanza - aprendizaje inclusivo y complementario a los cursos teóricos - prácticos. Una experiencia con estudiantes del curso física, https://doi.org/10.26507/rei.v11n22.602, Rev. Educación en Ingeniería, 11(22), 4-12, (2016). 
Calder, N., Using Scratch to Facilitate Mathematical Thinking, https://doi.org/10.15663/wje.v23i2.654, Waikato J. of Education, 23(2), 43-58, ( 2018).

Dapozo, G., Petris, R., y otros tres autores, Capacitación en programación para incorporar el pensamiento computacional en las escuelas, Rev. iberoam. tecnol. educ. educ. tecnol. 1(18), 194-203 (2016).

Girón, A., Objetivos del desarrollo sostenible y la agenda 2030:frente a las políticas públicas y los cambios de gobierno en américa latina, https://doi.org/10.1016/j.rpd.2016.08.001, Problemas del Desarrollo, 47(186), 3-8 (2016).

Hewett, K., Zeng, G. y Pletcher, B., The Acquisition of 21st-Century Skills Through Video Games: Minecraft Design Process Models and Their Web of Class Roles, https://doi.org/10.1177/1046878120904976, Simulation and Gaming, 50(3), 336364 (2020).

Kordaki, M., Diverse categories of programming learning activities could be performed within Scratch, https://doi.org/10.1016/j.sbspro.2012.05.267. J. Procedia - Social and Behavioral Sciences, 46, 1162-1166(2012).

Küster, I., y Castillo, V., Efectos de los videojuegos en las marcas emplazadas: la transmisión de emociones, https://doi.org/10.1016/S1138-1442(14)60008-5, Rev. Española de Investigación de Marketing ESIC, 16(1), 29-58 (2012)

Loaiza, O., y Hincapié, D., Un estudio de las brechas municipales en calidad educativa en Colombia: 2000-2012, https://doi.org/10.1016/j.espe.2016.01.001, Ensayos sobre Política Económica, 34(79), 3-20 (2016).

Marimuthu, M., and Govender, P., Perceptions of scratch programming among secondary school students in KwaZuluNatal, South Africa, https://doi.org/10.23962/10539/26112, The African J. of Information and Communication, 21(1), 51 80 (2018).

MEN, Estándares Básicos de Competencias en Lenguaje, Matemáticas, Ciencias y Ciudadanas. 1ํEd, 46-95, Ministerio de Educación Nacional, Bogotá (2016).

Moreno, J., Robles, G., y Román, M., Towards Data-Driven Learning Paths to Develop Computational Thinking with Scratch, https://doi.org/10.1109/TETC.2017.2734818, IEEE Transactions on Emerging Topics in Computing, 8(1), 193205 (2020).

Monjelat, N., Programación de tecnologías para la inclusión social con Scratch: Prácticas sobre el pensamiento computacional en la formación docente, https://doi.org/10.15359/ree.23-3.9, Rev. Educare,23(3) ,1409-4258 (2019).

Ouahbi, I., Kaddari, F., and other 3 autors, Learning Basic Programming Concepts by Creating Games with Scratch Programming Environment, https://doi.org/10.1016/j.sbspro.2015.04.224, Procedia - Social and Behavioral Sciences, 191(1), 1479-1482 (2015).

Pejman, M., and Lennart, N., Introducing the biometric storyboards tool for games user research, https://doi.org/10.1109/GEM.2014.7048098, J. IEEE, 1 (2014).

Pérez, A., Valdés, M., y otros dos autores, Estrategia didáctica para enseñar a planificar los procesos de enseñanza y aprendizaje de la matemática, https://doi.org/10.15517/revedu.v43i2.32236, Rev. Educación, 43(2), 1-18 (2019).

Rodríguez, J.A., González, J.A., and Sáez, J.M., Computational thinking and mathematics using Scratch: an experiment with sixth-grade students, https://doi.org/10.1080/10494820.2019.1612448, Interactive Learning Environments, 28(3),316327 (2020).

Rueda, R., y Franco, M., Políticas educativas de TIC en Colombia: entre la inclusión digital y formas de resistencia transformación social, https://doi.org/10.17227/pys.num48-7370, Rev. Pedagogía y Saberes, 48(1), 9-25 (2018).

Sánchez, I. I., Medina, F., y Rojas, F., El ingeniero de inclusión con videojuegos, https://doi.org/10.26507/rei.v10n19.532, Rev. Educación en Ingeniería, 10(9), 116-12. (2015).

Scherer, R., Siddiq, F., y Sánchez, B., A meta-analysis of teaching and learning computer programming: Effective instruc-tional approaches and conditions, https://doi.org/10.1016/j.chb.2020.106349, Computers In Human Behavior, 109, 1-18 (2020)

Thuneberg, H., Salmi, H., y Bogner, F., How creativity, autonomy and visual reasoning contribute to cognitive learning in a STEAM hands-on inquiry-based math module, https://doi.org/10.1016/j.tsc.2018.07.003, Thinking Skills and Creativity, 29(1), 153-160 (2018).

Vásquez, D., Políticas de inclusión educativa: una comparación entre Colombia y Chile, https://doi.org/10.5294/edu.2015.18.1.3, Rev. Pedagogía Social, 18(1), 45-61 (2015).

Vidal, C., Cabezas, C., y otros dos autores, Experiencias Prácticas con el Uso del Lenguaje de Programación Scratch para Desarrollar el Pensamiento Algorítmico de Estudiantes en Chile, Formación Universitaria, https://doi.org/10.4067/S0718-50062015000400004, Rev. Formación Universitaria, 8(4), $23-32$ (2015).

Zhang, L., and Nouri, J., A systematic review of learning computational thinking through Scratch in K-9, https://doi.org/10.1016/j. J. Computers \& Education,1, 1-34 (2019). 